\title{
Evaluation of Integrated Pest Management Modules against Brinjal Shoot and Fruit Borer Leucinodes orbonalis (Guenee) (Lepidoptera: Pyralidae)
}

\author{
Ch. Chinnabbai ${ }^{*}$, S. Dayakar ${ }^{2}$, A. Sujatha ${ }^{3}$, P. Anil Kumar ${ }^{4}$ and S.K. Nafeez Umar ${ }^{5}$ \\ ${ }^{1}$ Department of Entomology, College of Horticulture, Venkataramannagudem, India \\ ${ }^{2}$ Department of Entomology, Agricultural College, Rajamahendravaram, India \\ ${ }^{3}$ Department of Entomology, Dr. YSRHU, Venkataramannagudem, India \\ ${ }^{4}$ Department of Plant Pathology, ${ }_{5}^{5}$ Department of Statistics and Computer applications, \\ Agricultural College, Bapatla, India
}

*Corresponding author

\begin{abstract}
A B S T R A C T
An investigation was carried out during rabi 2017-18 to evaluate the bioefficacy of seven integrated pest management modules against shoot and

\section{Keywords}

Brinjal, shoot and fruit borer, IPM modules evaluation

\section{Article Info}

Accepted: 04 June 2019 Available Online: 10 July 2019 fruit borer, Leucinodes orbonalis (Guenee) in brinjal. M1-Moderately resistant brinjal genotype selected from screening experiments (IC 136061), M2Control, M3-M6 comprised of various components from cultural, physical, mechanical, biological and chemical control methods in various combinations, M7-farmers practice.Module-7 consisting farmers practice received least overall mean per cent fruit infestation (10.77\%) on number and weight basis compared to all other modules (M1-M6). However it was on par with M1 with regard to overall mean fruit infestation. Significantly superior performance in efficacy of modules in respect of fruit yield $(343.09 \mathrm{~kg} / \mathrm{plot}, 34309.33 \mathrm{~kg} / \mathrm{ha})$ was observed in M7 in comparison to other IPM modules M1 $(314.50 \mathrm{~kg} / \mathrm{plot}$, $31449.60 \mathrm{~kg} / \mathrm{ha})$, M2 (146.50 kg/plot, $14649.60 \mathrm{~kg} / \mathrm{ha})$, M3 (174.72 kg/plot, $17472.00 \mathrm{~kg} / \mathrm{ha})$, M4 (185.62 kg/plot, $18562.13 \mathrm{~kg} / \mathrm{ha})$, M5 (236.54 kg/plot, $23554.40 \mathrm{~kg} / \mathrm{ha})$, M6 (260.74 kg/plot, $26073.60 \mathrm{~kg} / \mathrm{ka})$.
\end{abstract}

\section{Introduction}

Brinjal is the most common and popular vegetable to all classes of people in India and other parts of the world. India is the second largest country after china in the world and accounts for about 11.89 MT with an area of $0.68 \mathrm{MH}$ under cultivation having productivity of $17.5 \mathrm{t} / \mathrm{ha}$. In Andhra Pradesh, it is grown in an area of $0.28 \mathrm{MH}$ with an annual production of 5.65 MT and productivity of $20.17 \mathrm{t} / \mathrm{ha}$ (NHB, Data base 2015).

Brinjal production is affected by many adverse factors and among them brinjal shoot and fruit borer, Leucinodes orbonalis Guenee has 
remained a major pest of brinjal (Haseeb et al., 2009). The yield loss caused by this pest has been estimated up to $67 \%$ in Bangladesh (Islam and Karim, 1991). This pest can cause a crop loss to the extent of $70 \%$ even after repeated insecticidal spray (Singh and Pandita, 2009). Synthetic insecticides are the most effective tools against this pest, however their indiscriminate use causes serious problems including pest resistance and environmental pollution (Panda and Khush, 1995). Toxic residues in harvested fruits cause serious health hazards to the consumers, and to the non-targeted organisms e.g., natural enemies and pollinators.

It is therefore necessary to develop and follow a rational approach with greater reliance on IPM to promote sustainability and to reduce the number of application of hazardous chemicals. In this regard, the present investigation was planned to evaluate some integrated pest management modules contained cultural, physical, mechanical, botanical, microbial, chemical control practices for the management of shoot and fruit borer.

\section{Materials and Methods}

The research trial was conducted at college farm, College of Horticulture, Dr. YSR Horticultural University, Venkataramannagudem during rabi 2017-18. Nursery with moderately resistant genotype selected from screening experiment (IC 136061) and Dommeru local brinjal variety was sown on raised beds. The trial was laid out in Randomized Blocked Design with a plot size $10 \mathrm{~m} \times 10 \mathrm{~m}$ with seven modules (treatments) and replicated thrice. The particulars and components in each module are given in Table 1. The border row crop (Sorghum) was sown 30 days prior to the transplantation of brinjal. The brinjal seedlings of 35 days old were transplanted at a spacing of $70 \mathrm{~cm} \times 60 \mathrm{~cm}$.
The intercrop coriander was sown after the transplantation of main crop. All recommended package of practices were followed to raise the crop, except plant protection measures. Insecticides were sprayed at 15 days interval in M4-M7. First spray was started at vegetative stage i.e. 35 DAT in M4 to M7. The subsequent sprays were applied at fifteen days interval.

The observations were recorded regarding shoot infestation, fruit infestation number basis, weight basis) and yield. The shoot and fruit infestations were recorded at 15 days interval by counting total number of healthy and infested shoot and fruits on five randomly selected and tagged plants in each module starting from 15 DAT to till final harvest for shoot infestation whereas from 45 DAT to till final harvest in case of fruit infestation.

The data on shoot infestation, fruit infestation (on number basis, weight basis) and fruit yield were analyzed statistically after suitable transformation for necessary parameters by following the standard procedure as suggested by Gomez and Gomez (1976). Mean per cent shoot infestation and per cent fruit infestation were calculated following the formula suggested by Wakil et al., (2009) while comparing the yield from different treatments, the per cent reduction in shoot infestation, per cent reduction in fruit infestation, per cent increase in yield over control were calculated by following the procedure given by Pradhan (1969).

Number of infested shoots

Per cent shoot infestation = ------------- $\mathrm{x} 100$

Total number of shoots

Per cent reduction in shoot infestation $=$

Per cent shoot $\quad-\quad$ Per cent shoot in infestation in treatment infestation in control $\mathrm{x} 100$

Per cent shoot infestation in treatment 
Per cent fruit Infestation (by number) $=$

Number of infested fruits $\mathrm{x} 100$

Total number of fruits

Per cent fruit Infestation (by weight) $=$

Weight of infested fruits

Weight of total fruits

Per cent fruit infestation in treatment Per cent fruit infestation in control

Per cent reduction

In fruit infestation = --------------------- x 100

Per cent fruit infestation in treatment

Per cent increase In yield over control $=$

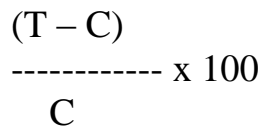

Where, $\mathrm{T}=$ Yield in treatment; $\mathrm{C}=$ Yield in control

\section{Results and Discussion}

\section{Bioefficacy of IPM modules on shoot infestation}

The overall mean percent shoot infestation data of all the modules presented in Table. 2. revealed that, M1 was very effective which recorded the lowest incidence of shoot infestation (12.98\%) followed by M7 (15.15\%), M6 (18.79\%), M5 (21.94\%), M4 (24.34\%), M3 (27.27\%) and M2 (30.42\%). M7 also showed significant variation in mean per cent shoot infestation and found superior to other modules (M2-M6) except M1. With the early onset of synthetic insecticide spray in M7 at 35DAT protected the plant from fruit borer infestation where as botanical spray in M4-M6 recorded with more fruit borer infestation in the early stages of fruit bearing. Significant reduction in per cent shoot infestation over control was observed in all
IPM modules, MI (61.10\%), M3 (10.08\%), M4 (20.46\%), M5 (28.64\%), M6 (37.48\%) and M7 (53.07\%). Module M1 was planted with the moderately resistant genotype (IC 136061), selected from the screening experiment expressed the Antixenosis mechanism of resistance through physicomorphic (Narrow shoot thickness, trichomes on leaf lamina, shoot) and biochemical factors (High content of Phenol, peroxidase, Phenylalanine Ammonialyase) and hence recorded with less shoot infestation without any plant protection measures compared to other modules (M3 - M6).

\section{Bioefficacy of IPM modules on fruit infestation (Number)}

The data on overall mean per cent fruit infestation on number basis (Table 3) in module M1 (15.08\%) was on par with module M7 (14.27\%). The efficacy of modules M6 (30.75\%), M5 (36.73\%), M4 (40.34\%), M3 $(46.52 \%)$ were significantly differed and found inferior to M1 and M7 in terms of mean per cent fruit infestation on number basis, but are comparatively superior over untreated control M2 (51.18\%). The rate of incidence of fruit borer infestation was more in M4 to M6 compared to M7 at 45 DAT. This is due to the initiation of insecticide spray at 10 DAT in M7 whereas in M4-M7 the spray schedule was initiated at 45 DAT. Significant reduction in per cent fruit infestation over control was observed in all IPM modules, MI (70.53\%), M3 (9.09\%), M4 (21.17\%), M5 (28.22\%), M6 (39.92\%), M7 (66.13\%).The inherited character acquired by the moderately resistant genotype through physicomorphic (Pedicel length, calyx length, fruit length, fruit diameter, fruit colour and fruit shape) and biochemical factors (High content of Phenol, peroxidase and Phenylalanine Ammonialyase) of fruit enabled the plant to avoid the fruit borer infestation through antixenosis mechanism. 
Table.1 Particulars of various IPM modules formulated against brinjal shoot and fruit borer, L.orbonalis

\begin{tabular}{|c|c|}
\hline Module & Particulars of the module \\
\hline M1 & $\begin{array}{l}\text { Moderately resistant genotype IC } 136061 \text { (selected from screening } \\
\text { experiment) }\end{array}$ \\
\hline M2 & Susceptible line (Untreated check) \\
\hline M3 & $\begin{array}{l}\text { Susceptible line }+ \text { Border crop }(\text { Sorghum })+\text { Intercrop }(\text { Brinjal- } \\
\text { Coriander } 2: 1)+ \text { Clipping and destruction of infested shoots from } 15 \\
\text { DAT }\end{array}$ \\
\hline M4 & $\begin{array}{l}\text { Susceptible line + Border crop (Sorghum) + Intercrop (Brinjal- } \\
\text { Coriander 2:1) + Clipping and destruction of infested shoots from } 15 \\
\text { DAT + Azadirachtin 1\% EC }(10000 \mathrm{ppm}) @ 3.0 \mathrm{ml} / 1 \text { (3sprays-35, } 65 \text {, } \\
95 \text { DAT) +Bacillus thuringiensis var kurustaki 5\% WP @ } 2 \mathrm{~g} / 1 \text { (3 } \\
\text { sprays- 50, 80,110 DAT) }\left(1 \times 10^{10} \mathrm{cfu} / \mathrm{gm}\right)\end{array}$ \\
\hline M5 & 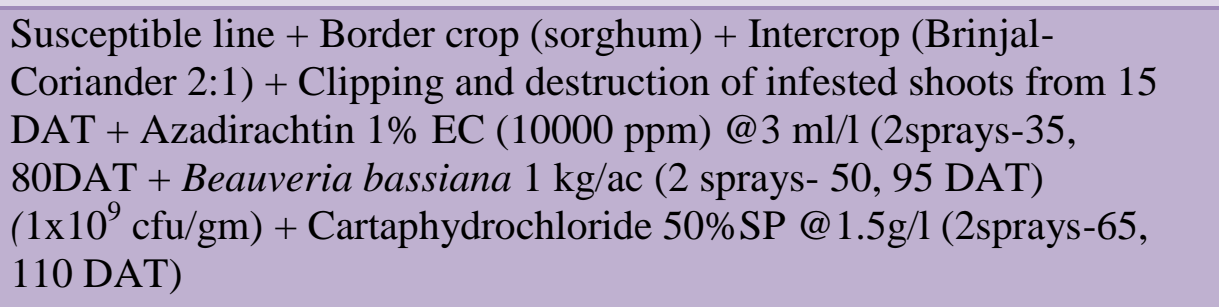 \\
\hline M6 & $\begin{array}{l}\text { Susceptible line + Border crop (Sorghum) + Intercrop (Brinjal- } \\
\text { Coriander 2:1) + Clipping and destruction of infested shoots from } 15 \\
\text { DAT + Azadirachtin 1\% EC (10000 ppm) } 3 \text { ml/l }(3 \text { sprays- 35, 65, } 125 \\
\text { DAT }+ \text { Spinosad 45\% SC @ } 0.36 \mathrm{ml} / 1 \text { (2 sprays- 50, 95 DAT })+ \\
\text { Cartaphydrochloride 50\% SP @ } 1.5 \mathrm{~g} / 1 \text { (2 sprays - 80,110 DAT) }\end{array}$ \\
\hline M7 & 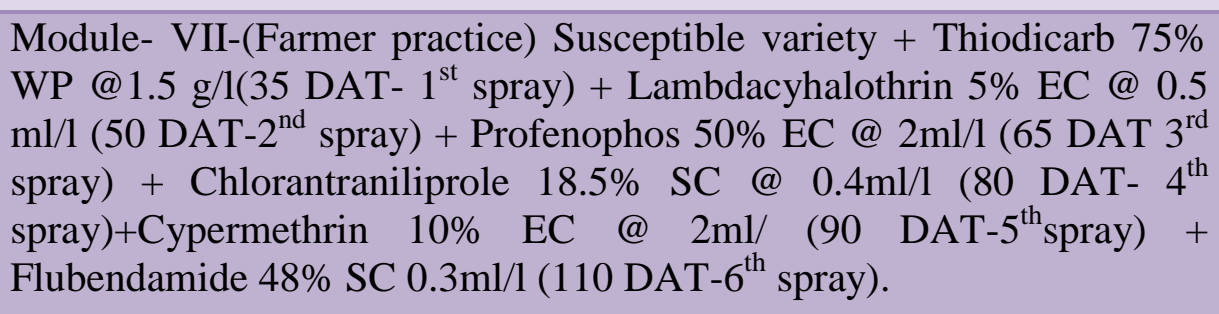 \\
\hline
\end{tabular}


Table.2 Evaluation of different IPM modules against brinjal shoot and fruit borer, L.orbonalis during rabi 2017-18

\begin{tabular}{|c|c|c|c|c|c|c|c|c|c|c|c|}
\hline \multirow[t]{2}{*}{ Module } & \multicolumn{10}{|c|}{ Mean per cent shoot infestation at 15 days interval } & \multirow{2}{*}{$\begin{array}{c}\text { Overall } \\
\text { Mean }\end{array}$} \\
\hline & 15 DAP & 30 DAP & $45 \mathrm{DAP}$ & $60 \mathrm{DAP}$ & 75 DAP & 90 DAP & 105 DAP & 120 DAP & 135 DAP & 150 DAP & \\
\hline M1 & $\begin{array}{c}30.00 * \\
(33.18) \mathrm{a}\end{array}$ & $\begin{array}{c}28.85 \\
(32.48) \mathrm{a}\end{array}$ & $\begin{array}{c}20.42 \\
(26.82) \mathrm{a}\end{array}$ & $\begin{array}{c}14.59 \\
(22.43) \mathrm{a}\end{array}$ & $\begin{array}{c}9.97 \\
(18.39) \mathrm{a}\end{array}$ & $\begin{array}{c}7.37 \\
(15.73) \mathrm{a}\end{array}$ & $\begin{array}{c}5.96 \\
(14.11) \mathrm{ab}\end{array}$ & $\begin{array}{c}5.68 \\
(13.77) \mathrm{ab}\end{array}$ & $\begin{array}{c}4.61 \\
(12.36) \mathrm{a}\end{array}$ & $\begin{array}{c}2.39 \\
(8.84) \mathrm{a}\end{array}$ & $\begin{array}{c}12.98 \\
(21.12) \mathrm{a}\end{array}$ \\
\hline M2 & $\begin{array}{c}52.22 \\
(46.27) \mathrm{d}\end{array}$ & $\begin{array}{c}47.04 \\
(43.3) \mathrm{c}\end{array}$ & $\begin{array}{c}49.05 \\
(44.45) \mathrm{d}\end{array}$ & $\begin{array}{c}37.95 \\
(38.01) \mathrm{d}\end{array}$ & $\begin{array}{c}29.15 \\
(32.66) \mathrm{c}\end{array}$ & $\begin{array}{c}23.85 \\
(29.23) \mathrm{e}\end{array}$ & $\begin{array}{c}23.47 \\
(28.96) \mathrm{f}\end{array}$ & $\begin{array}{c}18.94 \\
(25.79) \mathrm{e}\end{array}$ & $\begin{array}{c}11.81 \\
(20.09) \mathrm{c}\end{array}$ & $\begin{array}{c}11.42 \\
(19.63) \mathrm{d}\end{array}$ & $\begin{array}{c}30.42 \\
(33.47) \mathrm{g}\end{array}$ \\
\hline M3 & $\begin{array}{c}51.55 \\
(45.89)\end{array}$ & $\begin{array}{c}46.32 \\
(42.88) b c\end{array}$ & $\begin{array}{c}39.21 \\
(38.76) b c\end{array}$ & $\begin{array}{c}32.27 \\
(34.57) \mathrm{d}\end{array}$ & $\begin{array}{c}25.59 \\
(30.38) \mathrm{c}\end{array}$ & $\begin{array}{c}22.88 \\
(28.56) \mathrm{de}\end{array}$ & $\begin{array}{c}21.74 \\
(27.78) \mathrm{e}\end{array}$ & $\begin{array}{c}13.78 \\
(21.79) \mathrm{d}\end{array}$ & $\begin{array}{c}11.51 \\
(19.83) b c\end{array}$ & $\begin{array}{c}7.17 \\
(15.51) \mathrm{c}\end{array}$ & $\begin{array}{c}27.27 \\
(31.48) f\end{array}$ \\
\hline M4 & $\begin{array}{c}50.01 \\
(45.00) b\end{array}$ & $\begin{array}{c}40.06 \\
(39.26) b c\end{array}$ & $\begin{array}{c}36.43 \\
(37.08) b c\end{array}$ & $\begin{array}{c}29.96 \\
(33.16) c\end{array}$ & $\begin{array}{c}24.96 \\
(29.95) c\end{array}$ & $\begin{array}{c}20.09 \\
(26.63) \mathrm{cd}\end{array}$ & $\begin{array}{c}16.11 \\
(23.66) \mathrm{de}\end{array}$ & $\begin{array}{c}11.64 \\
(19.95) \mathrm{d}\end{array}$ & $\begin{array}{c}8.84 \\
(17.29) b c\end{array}$ & $\begin{array}{c}5.37 \\
(13.36) \mathrm{c}\end{array}$ & $\begin{array}{c}24.34 \\
(29.56) \mathrm{e}\end{array}$ \\
\hline M5 & $\begin{array}{c}50.01 \\
(45.01) b\end{array}$ & $\begin{array}{c}38.42 \\
(38.28) b c\end{array}$ & $\begin{array}{c}32.85 \\
(34.94) b c\end{array}$ & $\begin{array}{c}28.75 \\
(32.33) b c\end{array}$ & $\begin{array}{c}22.05 \\
(27.99) b c\end{array}$ & $\begin{array}{c}19.76 \\
(26.37) c\end{array}$ & $\begin{array}{c}11.75 \\
(20.00) \mathrm{cd}\end{array}$ & $\begin{array}{c}7.31 \\
(15.67) \mathrm{cd}\end{array}$ & $\begin{array}{c}4.92 \\
(12.81) \mathrm{ab}\end{array}$ & $\begin{array}{c}3.60 \\
(10.93) b\end{array}$ & $\begin{array}{c}21.94 \\
(27.93) d\end{array}$ \\
\hline M6 & $\begin{array}{c}48.87 \\
(44.35) b\end{array}$ & $\begin{array}{c}36.73 \\
(37.29) b\end{array}$ & $\begin{array}{c}31.31 \\
(33.98) b\end{array}$ & $\begin{array}{c}22.34 \\
(28.12) b\end{array}$ & $\begin{array}{c}19.28 \\
(25.83) b\end{array}$ & $\begin{array}{c}10.87 \\
(19.24) b\end{array}$ & $\begin{array}{c}7.56 \\
(15.96) c\end{array}$ & $\begin{array}{c}5.22 \\
(13.2) b c\end{array}$ & $\begin{array}{c}3.51 \\
(10.73) \mathrm{a}\end{array}$ & $\begin{array}{c}2.27 \\
(8.66) \mathrm{ab}\end{array}$ & $\begin{array}{c}18.79 \\
(25.69) \mathrm{c}\end{array}$ \\
\hline M7 & $\begin{array}{c}53.63 \\
(47.08) \mathrm{c}\end{array}$ & $\begin{array}{c}45.10 \\
(42.18) \mathrm{c}\end{array}$ & $\begin{array}{c}13.91 \\
(21.82) \mathrm{a}\end{array}$ & $\begin{array}{c}10.46 \\
(18.79) a\end{array}$ & $\begin{array}{c}6.51 \\
(14.58) \mathrm{a}\end{array}$ & $\begin{array}{c}5.26 \\
(13.02) \mathrm{a}\end{array}$ & $\begin{array}{c}4.83 \\
(12.43) \mathrm{a}\end{array}$ & $\begin{array}{c}3.13 \\
(10.1) \mathrm{a}\end{array}$ & $\begin{array}{c}2.16 \\
(8.43) \mathrm{a}\end{array}$ & $\begin{array}{c}1.38 \\
(6.65) \mathrm{ab}\end{array}$ & $\begin{array}{c}15.15 \\
(19.15) b\end{array}$ \\
\hline SEM \pm & 1.45 & 1.59 & 1.90 & 0.95 & 0.99 & 0.92 & 1.16 & 1.06 & 1.28 & 0.76 & 0.40 \\
\hline $\begin{array}{l}\text { CD } \\
(P=0.05)\end{array}$ & 4.47 & 4.91 & 5.86 & 2.94 & 3.07 & 2.85 & 3.57 & 3.27 & 3.96 & 2.35 & 1.23 \\
\hline $\mathrm{CV}(\%)$ & 5.74 & 6.95 & 9.43 & 5.34 & 6.63 & 7.13 & 10.02 & 10.60 & 15.36 & 11.11 & 2.52 \\
\hline
\end{tabular}

Mean of 5 plants

Values in the parentheses are arc sin transformed

Means followed by same alphabet do not differ significantly by DMRT at $5 \%$ 
Table.3 Evaluation of different IPM modules against brinjal shoot and fruit borer, L.orbonalis during rabi 2017-18

\begin{tabular}{|c|c|c|c|c|c|c|c|c|c|}
\hline \multirow[t]{2}{*}{ Module } & \multicolumn{8}{|c|}{ Mean per cent fruit infestation(number basis) at 15 days interval } & \multirow{2}{*}{$\begin{array}{c}\text { Overall } \\
\text { Mean }\end{array}$} \\
\hline & 45 DAP & 60 DAP & 75 DAP & 90 DAP & 105 DAP & 120 DAP & 135 DAP & 150 DAP & \\
\hline M1 & $\begin{array}{c}18.00^{*} \\
(25.08) \mathrm{a}\end{array}$ & $\begin{array}{c}17.23 \\
(24.34) \mathrm{a}\end{array}$ & $\begin{array}{c}15.32 \\
(22.87) \mathrm{a}\end{array}$ & $\begin{array}{c}14.70 \\
(22.54) \mathrm{a}\end{array}$ & $\begin{array}{c}12.97 \\
(21.08) \mathrm{a}\end{array}$ & $\begin{array}{c}16.50 \\
(23.88) \mathrm{a}\end{array}$ & $\begin{array}{c}13.24 \\
(21.31) \mathrm{a}\end{array}$ & $\begin{array}{c}12.66 \\
(20.72) \mathrm{a}\end{array}$ & $\begin{array}{c}15.08 \\
(22.83) \mathrm{a}\end{array}$ \\
\hline M2 & $\begin{array}{c}54.44 \\
(47.57) \mathrm{c}\end{array}$ & $\begin{array}{c}51.11 \\
(45.64) c\end{array}$ & $\begin{array}{c}52.77 \\
(46.59) \mathrm{d}\end{array}$ & $\begin{array}{c}50.00 \\
(45.00) \mathrm{c}\end{array}$ & $\begin{array}{c}56.66 \\
(48.83) \mathrm{d}\end{array}$ & $\begin{array}{c}54.44 \\
(47.57) \mathrm{d}\end{array}$ & $\begin{array}{c}44.44 \\
(41.8) \mathrm{d}\end{array}$ & $\begin{array}{c}48.88 \\
(44.35) \mathrm{c}\end{array}$ & $\begin{array}{c}51.18 \\
(45.67) \mathrm{f}\end{array}$ \\
\hline M4 & $\begin{array}{c}52.22 \\
(46.27) \mathrm{c}\end{array}$ & $\begin{array}{c}45.55 \\
(42.44) b c\end{array}$ & $\begin{array}{c}40.00 \\
(39.22) \mathrm{c}\end{array}$ & $\begin{array}{c}36.66 \\
(37.24) b\end{array}$ & $\begin{array}{c}43.89 \\
(41.47) \mathrm{c}\end{array}$ & $\begin{array}{c}40.00 \\
(39.22) \mathrm{c}\end{array}$ & $\begin{array}{c}34.44 \\
(35.86) \mathrm{c}\end{array}$ & $\begin{array}{c}30.00 \\
(33.18) b\end{array}$ & $\begin{array}{c}40.34 \\
(39.43) d\end{array}$ \\
\hline M5 & $\begin{array}{c}51.11 \\
(45.64) \mathrm{c}\end{array}$ & $\begin{array}{c}38.88 \\
(38.55) b\end{array}$ & $\begin{array}{c}37.22 \\
(37.58) \mathrm{c}\end{array}$ & $\begin{array}{c}37.22 \\
(37.54) b\end{array}$ & $\begin{array}{c}37.22 \\
(37.54) b c\end{array}$ & $\begin{array}{c}31.11 \\
(33.85) b\end{array}$ & $\begin{array}{c}28.88 \\
(32.45) b c\end{array}$ & $\begin{array}{c}32.22 \\
(34.54) b\end{array}$ & $\begin{array}{c}36.73 \\
(37.3) \mathrm{c}\end{array}$ \\
\hline SEM \pm & 1.086 & 1.747 & 1.687 & 1.458 & 1.593 & 1.657 & 1.335 & 1.967 & 0.683 \\
\hline $\mathrm{CD}(\mathrm{P}=0.05)$ & 3.347 & 5.384 & 5.199 & 4.493 & 4.908 & 5.107 & 4.115 & 6.062 & 2.105 \\
\hline $\mathrm{CV}(\%)$ & 4.586 & 8.336 & 8.628 & 7.327 & 7.947 & 8.432 & 7.198 & 10.807 & 3.391 \\
\hline
\end{tabular}

Mean of 5 plants

Values in the parentheses are arc sin transformed

Means followed by same alphabet do not differ significantly by DMRT at $5 \%$ 
Table.4 Evaluation of different IPM modules against brinjal shoot and fruit borer, L.orbonalis during rabi 2017-18

\begin{tabular}{|c|c|c|c|c|c|c|c|c|c|}
\hline \multirow[t]{2}{*}{ Module } & \multicolumn{8}{|c|}{ Mean Per cent fruit infestation(weight basis) at 15 days interval } & \multirow{2}{*}{$\begin{array}{c}\text { Overall } \\
\text { Mean }\end{array}$} \\
\hline & 45 DAP & 60 DAP & 75 DAP & 90 DAP & 105 DAP & 120 DAP & 135 DAP & $150 \mathrm{DAP}$ & \\
\hline M1 & $\begin{array}{l}17.10^{*} \\
(24.4) \mathrm{a}\end{array}$ & $\begin{array}{c}16.41 \\
(23.71) \mathrm{a}\end{array}$ & $\begin{array}{c}14.53 \\
(22.24) \mathrm{a}\end{array}$ & $\begin{array}{c}13.93 \\
(21.92) \mathrm{a}\end{array}$ & $\begin{array}{c}12.37 \\
(20.56) b\end{array}$ & $\begin{array}{c}15.68 \\
(23.24) \mathrm{a}\end{array}$ & $\begin{array}{c}12.54 \\
(20.72) b\end{array}$ & $\begin{array}{c}12.09 \\
(20.22) \mathrm{a}\end{array}$ & $\begin{array}{c}14.33 \\
(22.23) \mathrm{a}\end{array}$ \\
\hline M2 & $\begin{array}{c}56.95 \\
(49.03) \mathrm{d}\end{array}$ & $\begin{array}{c}50.38 \\
(45.22) \mathrm{c}\end{array}$ & $\begin{array}{c}51.78 \\
(46.02) \mathrm{d}\end{array}$ & $\begin{array}{c}48.94 \\
(44.39) \mathrm{c}\end{array}$ & $\begin{array}{c}55.36 \\
(48.08) \mathrm{f}\end{array}$ & $\begin{array}{c}53.69 \\
(47.13) \mathrm{e}\end{array}$ & $\begin{array}{c}43.80 \\
(41.44) \mathrm{e}\end{array}$ & $\begin{array}{c}48.33 \\
(44.04) \mathrm{d}\end{array}$ & $\begin{array}{c}51.15 \\
(45.66) \mathrm{f}\end{array}$ \\
\hline M3 & $\begin{array}{c}50.26 \\
(45.15) \mathrm{c}\end{array}$ & $\begin{array}{c}47.25 \\
(43.42) \mathrm{c}\end{array}$ & $\begin{array}{c}46.13 \\
(42.77) \mathrm{c}\end{array}$ & $\begin{array}{c}49.13 \\
(44.5) \mathrm{c}\end{array}$ & $\begin{array}{c}42.55 \\
(40.69) \mathrm{d}\end{array}$ & $\begin{array}{c}45.05 \\
(42.15) \mathrm{d}\end{array}$ & $\begin{array}{c}43.83 \\
(41.45) \mathrm{e}\end{array}$ & $\begin{array}{c}43.04 \\
(40.99) \mathrm{d}\end{array}$ & $\begin{array}{c}45.90 \\
(42.65) \mathrm{e}\end{array}$ \\
\hline M4 & $\begin{array}{c}54.45 \\
(47.57) \mathrm{cd}\end{array}$ & $\begin{array}{c}44.27 \\
(41.70) b c\end{array}$ & $\begin{array}{c}38.98 \\
(38.62) \mathrm{c}\end{array}$ & $\begin{array}{c}34.01 \\
(35.65) \mathrm{b}\end{array}$ & $\begin{array}{c}39.56 \\
(38.95) \mathrm{d}\end{array}$ & $\begin{array}{c}38.37 \\
(38.27) \mathrm{c}\end{array}$ & $\begin{array}{c}34.71 \\
(36.06) \mathrm{d}\end{array}$ & $\begin{array}{c}31.85 \\
(34.28) \mathrm{c}\end{array}$ & $\begin{array}{c}39.52 \\
(38.95) d\end{array}$ \\
\hline M5 & $\begin{array}{c}50.11 \\
(45.06) \mathrm{c}\end{array}$ & $\begin{array}{c}37.82 \\
(37.92) b\end{array}$ & $\begin{array}{c}36.13 \\
(36.93) \mathrm{c}\end{array}$ & $\begin{array}{c}36.27 \\
(36.97) b\end{array}$ & $\begin{array}{c}32.92 \\
(34.97) \mathrm{c}\end{array}$ & $\begin{array}{c}30.39 \\
(33.41) b\end{array}$ & $\begin{array}{c}28.17 \\
(31.99) \mathrm{cd}\end{array}$ & $\begin{array}{c}31.49 \\
(34.08) \mathrm{c}\end{array}$ & $\begin{array}{c}35.41 \\
(36.51) c\end{array}$ \\
\hline M6 & $\begin{array}{c}49.01 \\
(44.43) \mathrm{c}\end{array}$ & $\begin{array}{c}37.84 \\
(37.93) b\end{array}$ & $\begin{array}{c}23.10 \\
(28.54) b\end{array}$ & $\begin{array}{c}31.24 \\
(33.98) b\end{array}$ & $\begin{array}{c}29.76 \\
(33.03) \mathrm{c}\end{array}$ & $\begin{array}{c}23.1 \\
(28.54) b\end{array}$ & $\begin{array}{c}25.92 \\
(30.48) \mathrm{c}\end{array}$ & $\begin{array}{c}19.56 \\
(26.09) b\end{array}$ & $\begin{array}{c}29.94 \\
(33.16) b\end{array}$ \\
\hline M7 & $\begin{array}{c}32.28 \\
(34.6) b\end{array}$ & $\begin{array}{c}11.49 \\
(19.17) \mathrm{a}\end{array}$ & $\begin{array}{c}10.64 \\
(18.97) a\end{array}$ & $\begin{array}{c}10.27 \\
(18.68) \mathrm{a}\end{array}$ & $\begin{array}{c}10.58 \\
(18.87) \mathrm{a}\end{array}$ & $\begin{array}{c}11.79 \\
(20.06) \mathrm{a}\end{array}$ & $\begin{array}{c}9.64 \\
(18.07) \mathrm{a}\end{array}$ & $\begin{array}{c}10.11 \\
(18.49) \mathrm{a}\end{array}$ & $\begin{array}{c}13.35 \\
(21.42) \mathrm{a}\end{array}$ \\
\hline SEM \pm & 1.438 & 1.735 & 1.819 & 1.485 & 1.599 & 1.632 & 1.240 & 1.997 & 0.632 \\
\hline $\mathrm{CD}(\mathbf{P}=\mathbf{0 . 0 5})$ & 4.430 & 5.346 & 5.606 & 4.577 & 4.927 & 5.029 & 3.820 & 6.152 & 1.949 \\
\hline CV (\%) & 6.005 & 8.424 & 9.422 & 7.628 & 8.243 & 8.499 & 6.824 & 11.093 & 3.187 \\
\hline
\end{tabular}

Values in the parentheses are arc sin transformed

Means followed by same alphabet do not differ significantly by DMRT at $5 \%$ 
Table.5 Evaluation of different IPM modules against brinjal shoot and fruit borer, L.orbonalis during rabi 2017-18

\begin{tabular}{|c|c|c|c|c|c|c|c|c|c|c|}
\hline \multirow[t]{2}{*}{ Module } & \multicolumn{8}{|c|}{ Fruit yield $\left(\mathrm{kg} \mathrm{plot}^{-1}\right)$ at 15 days interval } & \multirow{2}{*}{$\begin{array}{c}\text { Total } \\
\text { Yield (kg } \\
\left.\text { plot }^{-1}\right)\end{array}$} & \multirow{2}{*}{$\begin{array}{c}\text { Yield } \\
\left(\mathrm{kg} \mathrm{ha}^{-1}\right)\end{array}$} \\
\hline & 45 DAP & 60 DAP & 75 DAP & 90 DAP & 105 DAP & 120 DAP & 135 DAP & $150 \mathrm{DAP}$ & & \\
\hline M1 & $25.98 * b$ & $38.18 \mathrm{c}$ & $46.59 \mathrm{~d}$ & $47.19 \mathrm{~d}$ & $45.99 \mathrm{~b}$ & $46.59 \mathrm{e}$ & $38.08 \mathrm{c}$ & $26.88 \mathrm{~cd}$ & $314.50 \mathrm{e}$ & $31449.60 \mathrm{e}$ \\
\hline M2 & $17.47 \mathrm{a}$ & $16.13 \mathrm{a}$ & $21.50 \mathrm{a}$ & $21.50 \mathrm{a}$ & $18.82 \mathrm{a}$ & $17.47 \mathrm{a}$ & $18.82 \mathrm{a}$ & $14.78 \mathrm{a}$ & $146.50 \mathrm{a}$ & $14649.60 \mathrm{a}$ \\
\hline M3 & $17.47 \mathrm{a}$ & $22.85 \mathrm{a}$ & $22.85 \mathrm{a}$ & $28.22 \mathrm{~b}$ & $22.85 \mathrm{a}$ & $22.85 \mathrm{~b}$ & $20.16 \mathrm{a}$ & $17.47 \mathrm{ab}$ & $174.72 \mathrm{~b}$ & $17472.00 \mathrm{~b}$ \\
\hline M4 & $20.16 \mathrm{ab}$ & $25.31 \mathrm{~b}$ & $25.54 \mathrm{ab}$ & $25.54 \mathrm{ab}$ & $24.19 \mathrm{a}$ & $22.10 \mathrm{ab}$ & $22.62 \mathrm{a}$ & $20.16 \mathrm{ab}$ & $185.62 \mathrm{~b}$ & $18562.13 \mathrm{~b}$ \\
\hline M5 & $20.16 \mathrm{ab}$ & $29.57 \mathrm{~b}$ & $32.26 \mathrm{bc}$ & $38.98 \mathrm{c}$ & $38.98 \mathrm{~b}$ & $30.91 \mathrm{c}$ & $24.19 \mathrm{a}$ & $21.50 \mathrm{bc}$ & $236.54 \mathrm{c}$ & $23654.40 \mathrm{c}$ \\
\hline M6 & $21.50 \mathrm{ab}$ & $28.22 \mathrm{~b}$ & $38.98 \mathrm{~cd}$ & $41.66 \mathrm{~cd}$ & $38.98 \mathrm{~b}$ & $36.29 d$ & $28.22 \mathrm{ab}$ & $26.88 \mathrm{~cd}$ & $260.74 d$ & $26073.60 d$ \\
\hline M7 & $33.60 \mathrm{c}$ & $41.66 \mathrm{c}$ & $45.70 \mathrm{~d}$ & $55.93 \mathrm{e}$ & $56.45 \mathrm{c}$ & $47.94 \mathrm{e}$ & $33.60 \mathrm{bc}$ & $28.22 \mathrm{~d}$ & $343.09 \mathrm{f}$ & $34309.33 \mathrm{f}$ \\
\hline SEM \pm & 2.11 & 2.37 & 2.86 & 2.08 & 2.76 & 1.52 & 2.87 & 1.68 & 3.92 & 391.97 \\
\hline $\mathrm{CD}(\mathrm{P}=\mathbf{0 . 0 5})$ & 6.52 & 7.31 & 8.82 & 6.40 & 8.49 & 4.68 & 8.84 & 5.19 & 12.08 & 1207.78 \\
\hline CV $(\%)$ & 16.40 & 14.32 & 14.86 & 9.72 & 13.57 & 8.22 & 18.72 & 13.10 & 2.86 & 2.86 \\
\hline
\end{tabular}

Mean of 5 plants

Means followed by same alphabet do not differ significantly by DMRT at $5 \%$

Table.6 Comparative performance of IPM modules over control during 2017-18-rabi season

\begin{tabular}{|c|c|c|c|c|c|c|c|}
\hline \multirow[t]{2}{*}{ Module } & \multirow{2}{*}{$\begin{array}{c}\text { Mean } \\
\text { percent } \\
\text { shoot } \\
\text { infestation }\end{array}$} & \multirow[t]{2}{*}{$\begin{array}{l}\text { Mean percent } \\
\text { fruit infestation }\end{array}$} & $\begin{array}{c}\text { Total yield } \\
\left(\mathrm{kg} \mathrm{ha}^{-1}\right)\end{array}$ & \multirow{2}{*}{$\begin{array}{c}\text { Percent } \\
\text { reduction in } \\
\text { shoot infestation } \\
\text { over control }\end{array}$} & \multirow{2}{*}{$\begin{array}{c}\text { Per cent } \\
\text { reduction in } \\
\text { fruit infestation } \\
\text { over control }\end{array}$} & \multirow{2}{*}{$\begin{array}{l}\text { Per cent } \\
\text { increase in } \\
\text { yield over } \\
\text { control }\end{array}$} & \multirow{2}{*}{$\begin{array}{c}\text { Increase in } \\
\text { yield over } \\
\text { control } \\
\left(\mathrm{kg} \mathrm{ha}^{-1}\right)\end{array}$} \\
\hline & & & & & & & \\
\hline M1 & 12.56 & 15.08 & 31449.60 & 61.10 & 70.53 & 53.42 & 7825.64 \\
\hline M2 & 32.28 & 51.18 & 14649.60 & 0.00 & 0.00 & 0.00 & 0.00 \\
\hline M3 & 29.03 & 46.53 & 17472.00 & 10.08 & 9.09 & 16.15 & 2366.47 \\
\hline M4 & 25.68 & 40.35 & 18562.13 & 20.46 & 21.17 & 21.08 & 3087.85 \\
\hline M5 & 23.04 & 36.74 & 23654.40 & 28.64 & 28.22 & 38.07 & 5576.84 \\
\hline M6 & 20.18 & 30.75 & 26073.60 & 37.48 & 39.92 & 43.81 & 6418.64 \\
\hline M7 & 10.85 & 17.33 & 34309.33 & 53.07 & 66.13 & 57.30 & 8394.43 \\
\hline
\end{tabular}


Rahman et al., (2009); Shanmugham et al., (2015) reported similar response in IPM modules against shoot and fruit borer.

\section{Bioefficacy of IPM modules on fruit infestation (weight)}

At 90 DAT, low level of fruit borer infestation was observed in M7 (10.27\%) which was on par with M1 (12.37\%) where as M7 and M1 showed significant difference in mean per cent fruit infestation on weight basis with M6 (31.24\%), M5 (36.27\%), M4 (34.01\%), M3 (49.13\%) and M2 (48.94\%). The same trend of reduction in the incidence of fruit infestation in different IPM modules was observed even at 135 DAP and the lowest incidence of $9.64 \%$ was recorded in M7 as against $43.80 \%$ in $\mathrm{M} 2$.

Overall mean per cent fruit infestation data on weight basis (Table 4) revealed that among the different IPM modules, M7 and M1 are on par with low fruit borer infestation (13.35\%, $14.33 \%)$ followed by M6 (29.94\%), M5 (35.41\%), M4 (39.52), M3 (45.90\%) and M2 $(51.15 \%)$. Both the modules showed significant difference in mean fruit infestation on weight basis with other modules (M2-M6). Rahman and Razzab Ali (2009); Shanmugham et al., (2015) reported similar response in IPM modules against fruit infestation on weight basis.

\section{Bioefficacy of IPM modules on fruit yield}

Data pertaining to marketable fruit yield of brinjal (Table 5) showed that all the treatments were effective and superior to the untreated check. The highest fruit yield of $34309.33 \mathrm{~kg} / \mathrm{ha}$ was recorded in M7 and M1 registered the next highest fruit yield $(31449.60 \mathrm{~kg} / \mathrm{ha})$ followed by M6 $(26073.60$ $\mathrm{kg} / \mathrm{ha})$, M5 (23654.40 kg/ha), M4 (18562.13 $\mathrm{kg} / \mathrm{ha}), \quad \mathrm{M} 3 \quad(17472.00 \mathrm{~kg} / \mathrm{ha})$ and M2 $(14649.60 \mathrm{~kg} / \mathrm{ha})$. Significant increase in per cent fruit yield over control was observed in all IPM modules, MI (53.42\%), M3 (16.15\%), M4 (21.08\%), M5 (38.07\%), M6 (43.81\%) and M7 (57.30\%). These findings are in collaboration with the findings of other workers (Chakraborti, 2001; Bhargava et al., 2003 and Mishra et al., 2004; Rahman and Razzab Ali, 2009; Rath and Maity, 2005; Shanmugham, et al., 2015) who conducted experiments with different IPM modules. Similar results were also obtained by various workers (Table 6).

It was concluded that brinjal shoot and fruit borer can be managed by selecting moderately resistant genotype along with timely application of cultural, mechanical, biological and chemical measures.

\section{References}

Bhargava, K. K., Sharma, H.S and Kaul, C.L. 2003. Bioefficacy efficacy insecticides against okra jassid and fruit borer. Pest Management and Economic Zoology. 9(2): 193-195.

Chakraborti, S. 2001. A biorational approach for the management of brinjal fruit and shoot borer, Leucinodes orbonalis Guen. Journal of Entomological Research. 25(1): 73-76.

Gomez, K.A. and Gomez, A.A. 1984. Statistical procedures for agricultural research (2 ed.). John wiley and sons, NewYork: 680.

Haseeb, M., Sharma, D. K and Qamar, M. 2009. Estimation of the losses caused by shoot and fruit borer, Leucinodes orbonalis Guen. (Lepidoptera: Pyralidae) in eggplant. Trends in Biological Sciences. 2 (1):68-69.

Islam, M. N and Karim, M. A. 1994. Integrated management of the brinjal shoot and fruit borer, Leucinodes orbonalis Guenee at Joydebpur. In: Ann. Res. Report, 1993-94. Entomol. 
Div., Bangladesh Agril. Res. Ins., Bangladesh. pp. 41-42.

Mishra, N. C., Ram, S., Swain, S.C and Rath, S. 2004. Effects of insecticides and bioproducts on shoot and fruit borer incidence in brinjal. Journal of Research, Birsa Agricultural Universirt. 16(2): 257-260.

NHB Data Base, 2015 published by National Horticultural Board. Department of Agriculture and Co-operation, Government of India.

Panda N and Khush G S. 1995. Host plant resistance to insects. $\mathrm{CAB}$ International in association with the International Rice Research Institute. 431p

Pradhan, S. 1969. Insect pests of crops. National Book Trust of India, New Delhi, pp 208.

Rahman, M. M. and Razzab Ali, Md. 2009. Evaluation of some selected options for managing brinjal shoot and fruit borer in two intensive brinjal growing areas. World Journal of Zoology. 4 (3): 169175.
Rath, L. K. and B. K. Maity (2005). Evaluation of a non-chemical IPM module for management of brinjal shoot and fruit borer. Journal of Applied and Zoological Research. 16(1): 3-4.

Shanmugam, P.S., Indhumathi, K., Vennila, M.A and Tamilselvan, N. 2015. Evaluation of biointensive pest management modules against brinjal shoot and fruit borer, Leucinodes orbonalis Guenee (Lepidoptera: Crambidae) under precision farming system. Pest Management in Horticultural Ecosystems. 21(2): 154158.

Singh P B and Pandita V K. 2009. Incidence of brinjal fruit and shoot borer in Haryana. Annals of Plant Protection Sciences. 17:145- 49.

Wakil, W., Ashfaq, M., Ghazanfar, M. U., Afzal, M. and Riasat, T. 2009. Integrated management of Helicoverpa armigera in chickpea in rainfed areas of Punjab, Pakistan. Phytoparasitica. 37: 415-420.

\section{How to cite this article:}

Chinnabbai, Ch., S. Dayakar, A. Sujatha, P. Anil Kumar and Nafeez Umar, S.K. 2019. Evaluation of Integrated Pest Management Modules against Brinjal Shoot and Fruit Borer Leucinodes orbonalis (Guenee) (Lepidoptera: Pyralidae). Int.J.Curr.Microbiol.App.Sci. 8(07): 211-220. doi: https://doi.org/10.20546/ijcmas.2019.807.027 